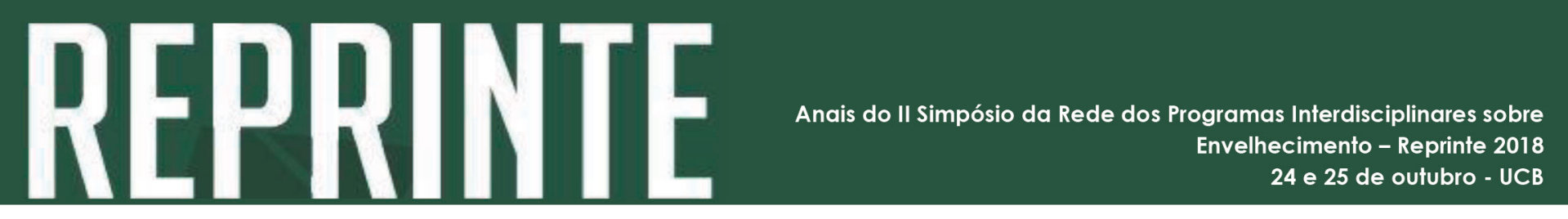

http://dx.doi.org/10.5335/rbceh.v16i1.10307

\title{
9) Socio-educational interventions as complementary strategies to improvement clinical parameters of Type 2 diabetes mellitus
}

\author{
Reis-Silva, D. ${ }^{1}$; Bertonceli, R. ${ }^{1}$; Batista, E. ${ }^{1}$; Feitosa-Bandeira, J. ${ }^{1}$; Cutrim-Lima, L. ${ }^{1}$; \\ Guimarães, V.V. ${ }^{1}$; Domingues M.A.R.C. ${ }^{1}$; Garay-Malpartida, H.M. ${ }^{1}$
}

\section{Introduction}

Type 2 diabetes mellitus (T2DM) is one of the most prevalent chronic diseases among the elderly, which impairs their functionality and the quality of life. Since high levels of social isolation are observed in the elderly population, we hypothesized that low social support network (SSN) could have negative effects in clinical evolution of T2DM. Therefore, the practice of socio educational activities could contribute to a favorable disease control and improvement of T2DM clinical parameters.

\section{Aims}

We evaluated, cross-sectionally and retrospectively, the relationship of SSN size with metabolic parameters of TD2M and the influence of socio-educational activities (i.e. crafts, jewelry, fitness, literacy, dance, guitar, singing, ballroom dancing, theater, yoga, flamenco, among others) in changes of the glycemic and lipid indexes in elderly patients ( $>60$ years old).

\section{Methods}

Six clinical parameters, fasting blood glucose (FBG), glycated hemoglobin (HbA1c), circulating triglycerides, HDL, LDL and total cholesterol levels were compared with social support network (SSN) which was measured in 89 T2DM patients by minimal map of relationship in older people (MMRI). The same parameters were compared between the group practicing socio educational activities $(\mathrm{n}=23)$ and non-practicing group $(\mathrm{n}=33)$. The chan-

School of Arts, Sciences and Humanities-University of São Paulo.Endereço para correspondência: R. Arlindo Béttio, 1000, Ermelino Matarazzo São Paulo - SP, Brasil. CEP: 03828-000. São Paulo, Brasil. Email: hgaray@gmail.com 
ges in values were established between two phases: 2001 to 2009 (before) and 2010 to 2011 (after), in both groups. The results were analyzed in two ways: a) vertical comparison of the averages of each individual clinical parameter; and b) horizontal comparison of total improvement rate (TIR), resulting from the sum of the individual improvement rate (iIR). To calculate iIR, clinical improvement received an arbitrary value (0.25) whereas no clinical improvement was ascribed a 0 value.

\section{Results}

We observed a discrete positive correlation between SSN size and glycemic control improvement of elderly T2DM patients. When socio-educational interventions are evaluated, we observed a significant improvement in $\mathrm{HbA} 1 \mathrm{c}$ levels of up $34.5 \%$ (odds ratio $=4.25 ; 95 \% \mathrm{CI}$, 1.17 - 15.45; $\mathrm{p}=0.0535$ ) in elderly diabetics practicing socio educational activities. However, the fasting glucose and total cholesterol values had no significant improvement. In the other hand, the horizontal analysis showed a significant difference between IMT means from elderly diabetics practicing socio educational activities when compared to the control group ( $\mathrm{p}=$ 0.03). It was observed a greater number of cases of clinical improvement over 50\% (IMT 0.75 and 1.0) in the socializing group (66\% vs $36 \%)$.

\section{Conclusions}

Our results suggested an apparent relationship between high SSN and practices of socio educational activities with significantly improvement of glicaemic control elderly T2DM patients. We speculated that enhancing social support affects direct or indirectly some health-related variables, although potential pathways activated by social and educational components to regulating glucose metabolism remains unclear. However, our data are suggesting that inclusion of socio-educational elements as non-pharmacological strategies for glicaemic control may contribute for an effective management of T2DM in public health. 